\title{
Snapshots of Anderson localization beyond the ensemble average
}

\author{
Ramy G. S. El-Dardiry, ${ }^{*}$ Sanli Faez, and Ad Lagendijk \\ FOM Institute AMOLF, Science Park 104, 1098 XG Amsterdam, The Netherlands
}

(Received 31 October 2011; revised manuscript received 25 June 2012; published 24 September 2012)

\begin{abstract}
We study $(1+1) D$ transverse localization of electromagnetic radiation at microwave frequencies directly by two-dimensional spatial scans. Since the longitudinal direction can be mapped onto time, our experiments provide unique snapshots of the buildup of localized waves. The evolution of the wave functions is compared with semianalytical calculations. Studies beyond ensemble averages reveal counterintuitive surprises. Oscillations of the wave functions are observed in space and explained in terms of a beating between the eigenstates.
\end{abstract}

DOI: 10.1103/PhysRevB.86.125132

PACS number(s): 42.25.Dd, 41.20.Jb, 72.15.Rn

\section{INTRODUCTION}

Recent years witnessed a renaissance in experimental studies on Anderson localization. This phenomenon, conceived by P. W. Anderson in $1958,{ }^{1}$ originally described the absence of diffusion of electrons in random lattices due to interference. Since Anderson localization is in essence a wave phenomenon, physicists have successfully extended the scope of localization studies to electromagnetic waves, ${ }^{2-5}$ ultrasound, ${ }^{6}$ and matter waves. $^{7-9}$

Similar to other phase-transition phenomena, dimensionality plays an important role. For $d \leqslant 2$, all states are localized, whereas for $d=3$ a phase transition from diffusive to localized behavior occurs at a critical scattering strength. ${ }^{10}$ In the special case of transverse localization, formulated by De Raedt et al., ${ }^{11}$ one dimension is designed not to be disordered, whereas disorder is introduced in the other dimension(s). As a consequence, waves spread out in the disorder-free dimension, but are confined in the other dimensions as long as the transverse system length $L$ is larger than the localization length $\xi$. In the paraxial limit transverse localization is described by an equation which closely resembles the time-dependent Schrödinger equation, ${ }^{11}$

$$
i \frac{\partial \psi}{\partial z}=\frac{1}{2 k n_{0}} H \psi,
$$

with $\psi$ the wave field and $k$ the vacuum wave number. The effective index of refraction is given by $n_{0}^{2} \equiv L^{-1} \int_{L} n^{2}(x) d x$. The Hamiltonian is defined as

$$
H \equiv \frac{\partial^{2}}{\partial x^{2}}+k^{2}\left[n^{2}(x)-n_{0}^{2}\right] .
$$

Effectively, transverse localization thus reduces the number of spatial coordinates in the system, since the coordinate along which the sample is extruded can be seen as the time axis in the time-dependent Schrödinger equation.

Pivotal experiments on weakly scattering disordered photonic lattices ${ }^{12,13}$ have focused on the observation of ensemble averaged intensity profiles after a certain fixed propagation distance. However, due to the very low refractive index contrast $\left(\sim 10^{-4}\right),{ }^{12}$ these studies did not reach the regime in which the spatial extent of the wave functions is fully arrested by disorder even after propagating over thousands of wavelengths. To make sure the localization length has truly converged in a transverse localization scheme requires measurements of the intensity along the disorder-free dimension. Very recently, transverse localization was experimentally put forward as a robust mechanism for optical waveguiding in fibers. ${ }^{14}$ For this type of application, knowledge on transverse localization beyond its ensemble averaged properties and as a function of propagation distance are prerequisites.

The central topic of this paper is therefore to measure and understand the evolution of transverse localized waves along the disorder-free dimension. After showing that our samples are indeed in the localizing regime by measuring over an ensemble of samples, we focus on the remarkable dynamics of a typical localized wave in a single realization. Counterintuitive nonstationary behavior of localized waves is observed, which we explain by decomposing these waves into the system's eigenstates semianalytically.

\section{EXPERIMENTAL METHODS}

In order to map the evolution of waves in a transverse localization sample, we scan a microwave antenna over the sample of interest using a stepper motor and a home-built scanning stage. The microwave transmission spectrum between this scanning antenna and a source antenna is measured using a vector network analyzer (Rhode and Schwartz ZVA 67). Figure 1 shows a sketch of the experimental apparatus.

Samples were fabricated by placing nylon bars $(3 \mathrm{~mm} \times$ $10 \mathrm{~mm} \times 1000 \mathrm{~mm})$ on top of an oxygen-free copper plate $(500 \mathrm{~mm} \times 1000 \mathrm{~mm})$. These nylon bars $[n=1.73$ (Ref. 15) $]$

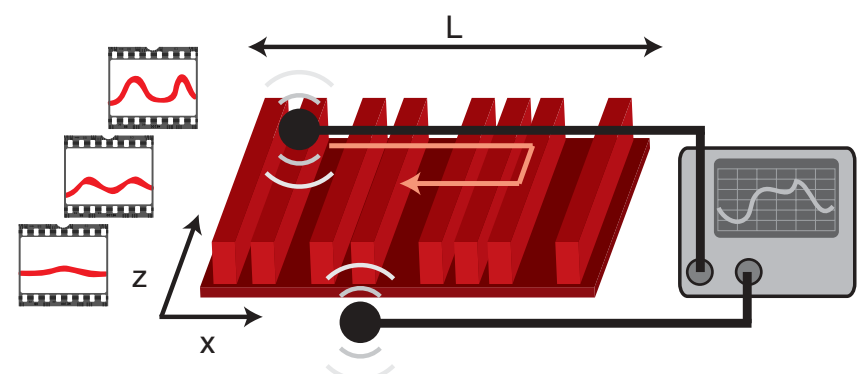

FIG. 1. (Color online) Experimental setup. Nylon bars (red) are placed on top of an oxygen-free copper plate, the distance between the bars is random in the transverse $x$ direction. The $z$ direction is disorder free. One of the two microwave antennas (black disks) is scanned over the sample and a vector network analyzer is used to measure the transmitted spectrum between the two antennas. Each scan along the $x$ axis is equivalent to a snapshot in time. 
are the scatterers in our system. Disorder was introduced into the system by varying the spacing between the nylon bars. The spacings were chosen randomly from a Poissonian distribution with a mean of $10 \mathrm{~mm}$. Introducing Poissonian disorder ensures that the presence of stop band effects is negligible. ${ }^{16}$ In addition, ordered samples were prepared with a lattice spacing of $20 \mathrm{~mm}$ in which clear stop bands were observed and calculated around 7 and $13 \mathrm{GHz}$. Styrofoam spacers ensured parallel alignment of the nylon bars.

\section{RESULTS}

The propagation of waves within an ordered and a disordered sample is shown in Fig. 2. The excitation frequency was set at $9.2 \mathrm{GHz}$, that is, outside any of the stop gaps of the ordered sample. The data were normalized for every row in the $x z$ plane to enhance the visibility of the wave function far away from the source. In the ordered sample, Fig. 2(a), waves spread out ballistically as a function of propagation distance. However, for the disordered sample, Fig. 2(b), the wave propagation is different: the wave initially spreads out, but at a certain stage stays confined to a bounded region. This type of two-dimensional spatial scans provides us with exceptional data for analyzing transverse localization in unprecedented detail.

In order to quantify the transverse confinement of wave intensity as a function of propagation distance, we calculate the inverse participation length (IPL). ${ }^{17}$ The IPL for a onedimensional intensity distribution $I(x)$ is defined as

$$
P(z) \equiv \frac{\int I^{2}(x, z) d x}{\left(\int I(x, z) d x\right)^{2}}
$$

and has a unit of inverse length. The IPL is inversely proportional to the spread of the wave function: a homogeneously extended wave spread out over the entire sample length $L$ leads to an IPL of $1 / L$. The IPL is insensitive to homogeneous losses that are possibly present in our semiopen system. To obtain a reliable value for the spread of wave functions, the ensemble averaged intensity profiles were determined by averaging over 20 realizations of disorder.

Figure 3 shows how the inverse of the IPL develops with increasing propagation distance for both the ordered sample and the ensemble of disordered samples at $9.2 \mathrm{GHz}$. In agreement with the qualitative picture we obtained from Fig. 2, we see that the extent of the wave function given by the inverse
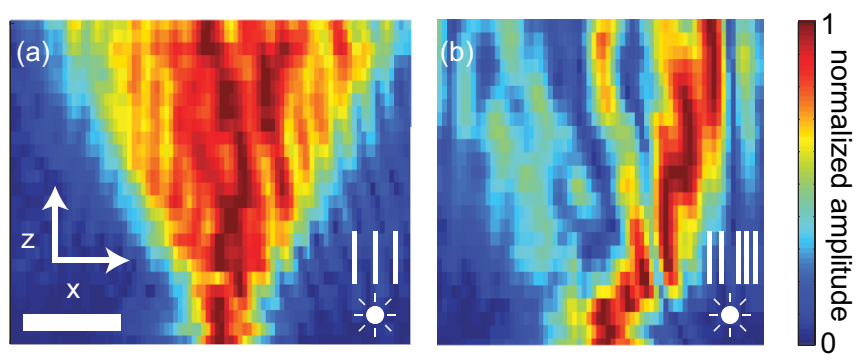

FIG. 2. (Color online) Experimentally determined false color images of the amplitude distribution for (a) an ordered and (b) a disordered sample at $9.2 \mathrm{GHz}$. Every row is normalized independently. The scale bar denotes $100 \mathrm{~mm}$.

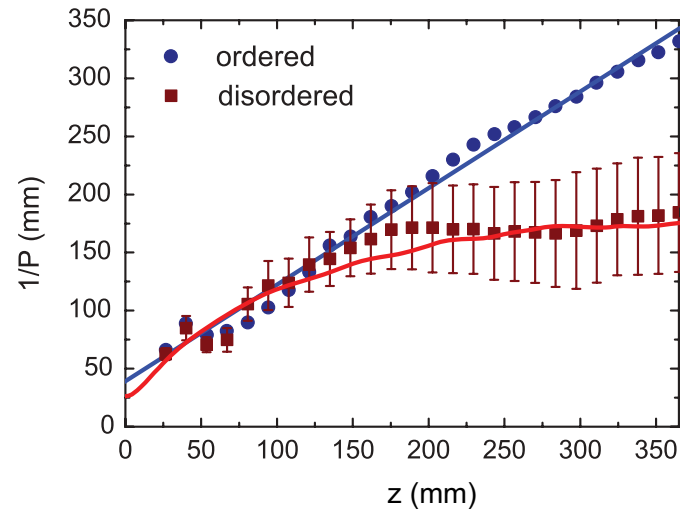

FIG. 3. (Color online) Participation ratio versus propagation distance at $9.2 \mathrm{GHz}$ for an ordered sample (blue) and a disordered ensemble (red). Red line: calculation for an ensemble of 100 disordered samples. Blue line: linear fit.

of the IPL increases linearly for the ordered sample. For the disordered ensemble, on the other hand, the IPL flattens off after a certain propagation distance. This settling of the IPL to a finite value constitutes a first direct experimental observation of the spatial evolution of transversely localized waves and ensures that we are truly in the transverse localizing regime.

After having studied the ensemble averaged properties of our system, we now aim to understand the propagation of waves for single realizations of disorder. What does a transverse localized wave look like? How does it evolve as a function of propagation distance? And how does it depend on the method of excitation? The answers to these questions simply cannot be given by focusing on an ensemble perspective, yet they are crucial for a complete understanding and possible application of the transverse localization phenomenon.

Figure 4(a) shows the first surprise we encountered when studying localized waves in a single realization of disorder. As shown in Fig. 4(a), we observe a periodic dynamics of the wave propagation. This oscillation contrasts to the general picture of localization as a "frozen" wave. ${ }^{18,19}$

In a second experiment we set out to determine the excitation dependence of the localized waves. In Fig. 5(a), we plot the spatial profile for 17 different excitation positions in one sample after $365 \mathrm{~mm}$ of propagation. Based on Fig. 3, this distance ensures that we are truly looking at localized waves. One might expect for a localizing sample clearly isolated regions of higher intensity that are independent on the position of excitation. Such patterns would appear as vertical stripes in Fig. 5(a). However, the measured spatial patterns of the isolated regions along the transverse dimension $x$ turn out to be strongly dependent on the position of the excitation antenna. To a large extent the detected radiation follows the position of the excitation antenna as indicated by the white diagonal, but some patterns appear to be antidiagonal.

\section{MODEL}

In order to build a basis for understanding the ensemble averaged data and the remarkable excitation dependence of localized waves in single realizations of disorder, the system is analyzed semianalytically. The solutions to Eq. (1) can 

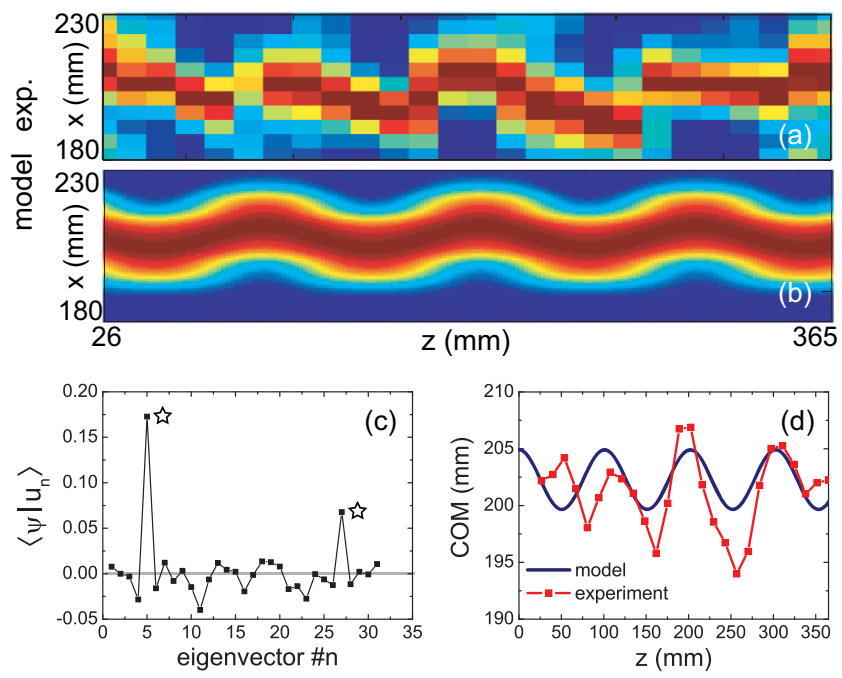

FIG. 4. (Color online) (a) Experimental and (b) calculated oscillations in the intensity profile for one sample excited at $10.2 \mathrm{GHz}$. Every column is normalized independently. (c) Expansion coefficients for the different eigenstates. Only two eigenstates, indicated by the stars, contribute significantly. (d) Center of mass of the intensity versus propagation direction for both the calculated and experimental data shown in (a) and (b).

be written as a linear combination of the Hamiltonian's eigenstates $u_{n}(x)$ :

$$
\psi(x, z)=\sum_{n} c_{n} u_{n}(x) \exp \left(-i \lambda_{n} z\right),
$$

where $\lambda_{n}$ is the eigenvalue belonging to eigenstate $u_{n}$ and $c_{n}$ is the $n$th expansion coefficient given by $c_{n}=\left\langle\psi \mid u_{n}\right\rangle$. The eigenvectors having eigenvalues closest to zero localize the easiest and correspond with eigenvectors with a small $k_{x}$ component.

The eigenstates and eigenvalues are calculated by diagonalizing the Hamiltonian matrix. The diagonal of the matrix contains the potential $k^{2}\left[n^{2}(x)-n_{0}^{2}\right]$ and the derivative in $x$ is approximated by using central differences creating a tridiagonal matrix when assuming absorbing boundary conditions. In principle, diagonalizing a $N \times N$ matrix results in $N$ eigenvalues and eigenvectors. However, most of these eigenvectors contain too high spatial frequencies in the transverse dimension, $k_{x}>k$, that are not excitable in our system. As a result we end up with only 30 eigenstates that obey the relation $k_{x} \leqslant k$. This number is determined by the spatial size of the system. The initial wave at $z=0$ is modeled as a Gaussian with a width of $1.15 \mathrm{~cm}$ given by the aperture of the excitation antenna. To compare the numerical calculation with experiment, we convolved the intensity of the calculated wave function with the aperture of the detection antenna

A decomposition into the system eigenstates for the sample in Fig. 4 reveals that just two eigenstates contribute significantly to the wave function as shown in Fig. 4(c). Using only these two eigenstates and their corresponding eigenvalues, we calculated the $z$ development of the wave function in Fig. 4(b) and compared them directly with experiment in Fig. 4(d). The calculated oscillations are quantitatively similar to those

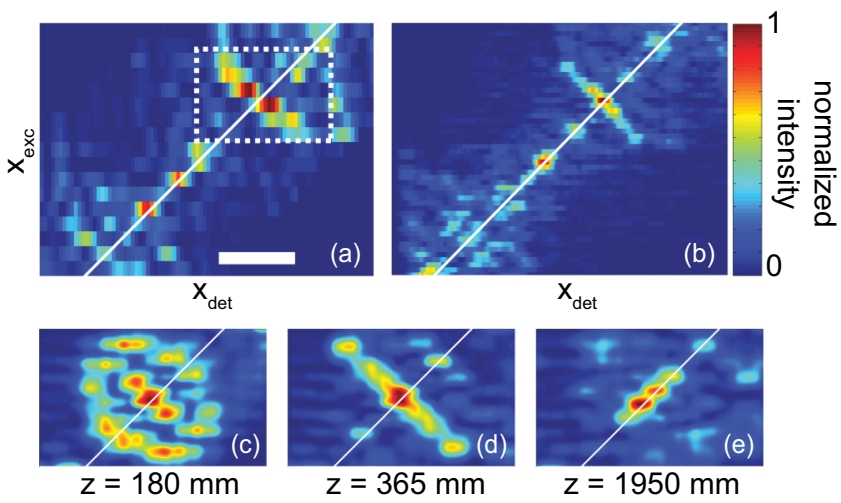

FIG. 5. (Color online) (a) Experimental and (b) numerically calculated false color plots of the wave function intensity in transverse direction after $365 \mathrm{~mm}$ of propagation along the $z$ direction for different positions of the excitation antenna at $9.2 \mathrm{GHz}$. The white lines indicate the position of the excitation antenna. The dashed box marks an antidiagonal wave profile. Scale bar denotes $100 \mathrm{~mm}$. (c)-(e) Calculations using mode decomposition for the area marked with the dashed box in (a) for 180, 365, and $1950 \mathrm{~mm}$ of propagation, respectively. Beating of eigenmodes can result in (c) circular, (d) antidiagonal, or (e) diagonal patterns.

observed in experiment. In general, the number of significantly contributing eigenvectors is often higher than 2 , which makes the beating less visible.

Motivated by the experimentally observed and unforeseen excitation dependence of the wave functions, we also calculated the excitation-detection patterns. In Fig. 5(b), it is shown that the position and shape of these patterns correspond with the measurements. The antidiagonal shapes are also clearly present in our numerical calculation, indicating that they are not caused by spurious effects such as mode perturbation due to the proximity of the receiver antenna.

In fact, the antidiagonal patterns shown in Figs. 5(a) and 5(b) are another observable consequence of the beating between the system's eigenstates. Depending on the accumulated phase during propagation, these antidiagonal excitationdetection patterns can become circular or diagonal as shown in Figs. 5(c)-5(e). The patterns are to a large extent point symmetric which originates from a flip in sign of the expansion coefficients when the excitation antenna crosses the central position of the beating oscillation.

\section{CONCLUSION AND DISCUSSION}

By using a disordered array of open waveguides, we have effectively measured the temporal dynamics of transverse localized waves from the moment of excitation until its complete halt. This observation is made possible by two important aspects of our setup: (1) mapping one spatial coordinate along the waveguides onto time in the corresponding wave-equation, and (2) the near field access to the wave function. We have observed, and confirmed by theoretical modeling, the beating between the localized eigenstates in time. Our observations prove that in a disordered system all localized states can be singled out as the true eigenstate of the system and their dynamics be measured individually. The localized eigenmodes 
have been isolated, and the eigenstates are identified based on their phase dynamics in an open system while they propagate, rather than their spectral line in the stationary regime. ${ }^{5}$ This enhanced control over the selective measurement of Anderson localized states enables us to study the nontrivial dynamics of them beyond the ensemble average.

We stress that the observed beatings and coherent mixing of localized waves is a general consequence of exciting a multimode system with an initial condition other than one of its eigenmodes. However, the modes of a strongly disordered optical system are peculiar in the sense that they have almost no direct relation with the form of the local potential and can only be solved when the full Hamiltonian is decomposed into its eigenfunctions. Therefore a method like ours that enables direct measurement of the modes will be very important in studies where quantum sources are interacting with Anderson localized modes. ${ }^{20}$
Since the transverse localization scheme allows for measuring snapshots of wave functions in time, it is a very convenient tool for studying the effect of different forms of disorder on wave propagation as put forward by recent work on photonic quasicrystals. ${ }^{21,22}$ Our work suggests that transverse localization can also be an excellent platform for studying the influence of perturbations and partial incoherence on localization. ${ }^{23}$

\section{ACKNOWLEDGMENTS}

We thank B. Gjonaj and P. Scalia for stimulating discussions and L. Kuipers for carefully reading the manuscript. This work is part of the research program of the Stichting voor Fundamenteel Onderzoek der Materie (FOM), which is financially supported by the Nederlandse Organisatie voor Wetenschappelijk Onderzoek (NWO). *dardiry@amolf.nl; http://www.randomlasers.com

${ }^{1}$ P. W. Anderson, Phys. Rev. 109, 1492 (1958).

${ }^{2}$ P. W. Anderson, Philos. Mag. B 52, 505 (1985).

${ }^{3}$ S. John, Phys. Rev. Lett. 58, 2486 (1987).

${ }^{4}$ A. A. Chabanov, M. Stoytchev, and A. Z. Genack, Nature (London) 404, 850 (2000).

${ }^{5}$ J. Wang and A. Z. Genack, Nature (London) 471, 345 (2011).

${ }^{6}$ H. Hu, A. Strybulevych, J. H. Page, S. E. Skipetrov, and B. A. van Tiggelen, Nat. Phys. 4, 945 (2008).

${ }^{7}$ J. Billy, V. Josse, Z. Zuo, A. Bernard, B. Hambrecht, P. Lugan,

D. Clément, L. Sanchez-Palencia, P. Bouyer, and A. Aspect, Nature (London) 453, 891 (2009).

${ }^{8}$ G. Roati, C. D'Errico, L. Fallani, M. Fattori, C. Fort, M. Z. G. Modugno, M. Modugno, and M. Inguscio, Nature (London) 453, 895 (2009).

${ }^{9}$ S. S. Kondov, W. R. McGehee, J. J. Zirbel, and B. DeMarco, Science 334, 66 (2011).

${ }^{10}$ E. Abrahams, P. W. Anderson, D. C. Licciardello, and T. V. Ramakrishnan, Phys. Rev. Lett. 42, 673 (1979).

${ }^{11}$ H. De Raedt, A. Lagendijk, and P. de Vries, Phys. Rev. Lett. 62, 47 (1989).

${ }^{12}$ T. Schwartz, G. Bartal, S. Fishman, and M. Segev, Nature (London) 446, 52 (2007).
${ }^{13}$ Y. Lahini, A. Avidan, F. Pozzi, M. Sorel, R. Morandotti, D. N. Christodoulides, and Y. Silberberg, Phys. Rev. Lett. 100, 013906 (2008).

${ }^{14}$ S. Karbasi, C. Mirr, P. G. Yarandi, A. Mafi, R. Frazier, and K. Koch, Opt. Lett. 37, 2304 (2012).

${ }^{15}$ Hewlett-Packard, Measuring Dielectric Constant with the HP 8510 Network Analyzer (Hewlett-Packard, Pablo Alto, CA, 1985).

${ }^{16}$ M. Rechtsman, A. Szameit, F. Dreisow, M. Heinrich, R. Keil, S. Nolte, and M. Segev, Phys. Rev. Lett. 106, 193904 (2011).

${ }^{17}$ Y. V. Fyodorov and A. D. Mirlin, Phys. Rev. Lett. 71, 412 (1993).

${ }^{18} \mathrm{~S}$. John, Nature (London) 390, 661 (1997).

${ }^{19}$ J. Chabé, G. Lemarié, B. Grémaud, D. Delande, P. Szriftgiser, and J. C. Garreau, Phys. Rev. Lett. 101, 255702 (2008).

${ }^{20}$ Y. Lahini, Y. Bromberg, D. N. Christodoulides, and Y. Silberberg, Phys. Rev. Lett. 105, 163905 (2010).

${ }^{21}$ Y. Lahini, R. Pugatch, F. Pozzi, M. Sorel, R. Morandotti, N. Davidson, and Y. Silberberg, Phys. Rev. Lett. 103, 013901 (2009).

${ }^{22}$ L. Levi, M. Rechtsman, B. Freedman, T. Schwartz, O. Manela, and M. Segev, Science 332, 1541 (2011).

${ }^{23}$ D. Čapeta, J. Radić, A. Szameit, M. Segev, and H. Buljan, Phys. Rev. A 84, 011801 (2011). 